\title{
Mésothéliome paratesticulaire malin : cas clinique et revue de la littérature
}

\author{
Imad Ziouziou, MD; Tariq Karmouni, MD; Khalid El Khader, MD; Abdellatif Koutani, MD; \\ Ahmed Iben Attya Andaloussi, MD
}

Service d'urologie B, CHU Ibn Sina, Faculté de médecine et de pharmacie, Université Mohamed V, Rabat, Morocco

Cite as: Can Urol Assoc J 2015;9(7-8):E565-7. http://dx.doi.org/10.5489/cuaj.2843 Published online August 10, 2015.

\section{Résumé}

Les mésothéliomes sont des tumeurs rares, liées surtout à l'exposition à l'amiante. La localisation dans la tunique vaginale est moins fréquente que les localisations pleurale et péritonéale. Nous faisons état du cas clinique d'un jeune patient de 30 ans qui présentait une masse testiculaire gauche avec lésion tissulaire intratesticulaire visible à l'échographie. Il a subi une orchidectomie ; I'examen anatomopathologique de la pièce opératoire orientait le diagnostic en faveur d'un mésothéliome malin de la tunique vaginale. Une hémiscrotectomie a été réalisée par la suite, et le bilan d'extension n'a pas révélé de lésion secondaire. L'évolution s'est avérée favorable moyennant un recul de quatre ans. À travers ce cas clinique et par l'entremise d'une revue de littérature, nous analysons les aspects épidémiologiques, diagnostiques et thérapeutiques de cette pathologie rare.

\section{Introduction}

Les mésothéliomes sont des tumeurs rares, dont la plupart sont liées à l'exposition à I'amiante (risque relatif de 1,34 par rapport au reste de la population). La localisation la plus fréquente est la plèvre, suivie de la cavité péritonéale et de la tunique vaginale ${ }^{1}$.

La majorité des mésothéliomes paratesticulaires se développent à partir de la tunique vaginale. Cependant, des cas de mésothéliomes primaires du cordon spermatique $(<10 \%)$ et de l'épididyme ont aussi été signalés ${ }^{1}$.

Décrit pour la première fois par Barbera et Rubino en $1957^{2}$, le mésothéliome de la tunique vaginale représente de $0,3 \%$ à 1,4\% de tous les mésothéliomes malins. II existe, à notre connaissance, environ 250 cas signalés dans la littérature ${ }^{3}$.

Nous faisons état, dans cet article, d'un mésothéliome de la tunique vaginale du testicule gauche chez un jeune patient de 30 ans et analysons, par l'entremise d'une revue de la littérature, les aspects épidémiologiques, diagnostiques et thérapeutiques de cette pathologie rare.

\section{Observation}

Monsieur A. M., âgé de 30 ans, sans antécédent pathologique notable ni exposition professionnelle à l'amiante, avait consulté pour une bourse gauche volumineuse lui occasionnant des douleurs gênant la marche et évoluant depuis 3 mois. À l'examen clinique, une masse scrotale dure de $4 \mathrm{~cm}$ a été décelée au niveau du pôle supérieur du testicule gauche, avec conservation du sillon épididymo-testiculaire (signe de Chevassu positif). L'échographie a mis en évidence, au niveau du pôle supérieur du testicule gauche, une masse tissulaire hypoéchogène arrondie de $3 \mathrm{~cm}$ évoquant une tumeur testiculaire (fig. 1). Par ailleurs, il n'y avait pas $\mathrm{d}^{\prime}$ hydrocèle, et le testicule controlatéral était sans anomalie. Les marqueurs tumoraux (alpha-fœtoprotéine, HCG, LDH) étaient normaux. Le patient a subi une orchidectomie par voie inguinale dont les suites ont été simples. L'examen anatomopathologique orientait le diagnostic en faveur d'un mésothéliome malin de la tunique vaginale (fig. 2). La tomodensitométrie thoraco-abdomino-pelvienne $\mathrm{n}^{\prime} \mathrm{a}$ pas mis en évidence de localisation pleurale associée ni de localisation secondaire. Une hémiscrotectomie a été réalisée, mais aucune tumeur n'a été décelée à l'examen anatomopathologique. Depuis, on a procédé annuellement à une tomodensitométrie thoraco-abdomino-pelvienne. L'évolution s'est avérée favorable, sans récidive locorégionale ni récidive à distance. Le recul est de quatre ans.

\section{Discussion}

Les mésothéliomes paratesticulaires sont des tumeurs rares qui touchent des patients de tout âge, avec un pic de fréquence de 55 à 75 ans. Seulement $10 \%$ des patients sont âgés de moins de 25 ans $^{4}$. 


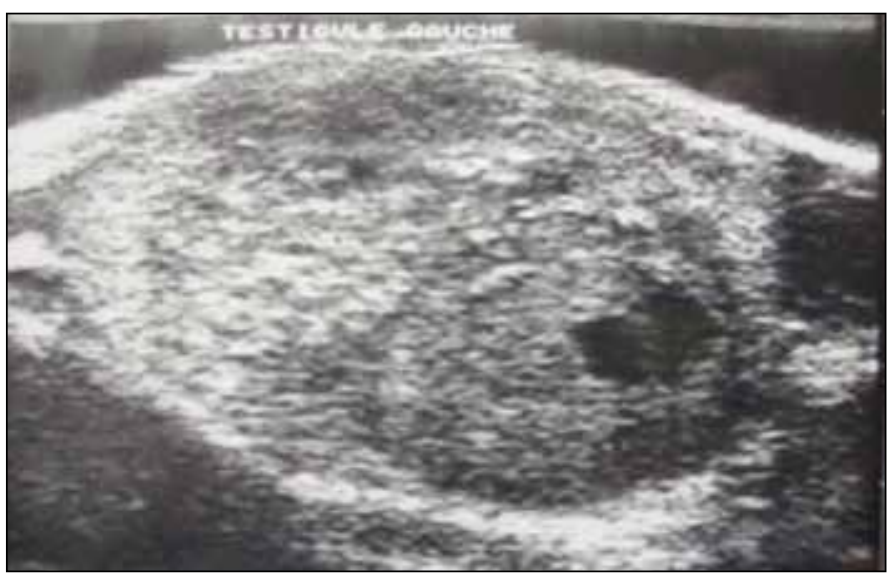

Fig. 1. Image échogène ovalaire de $3 \mathrm{~cm}$ de plus grand axe avec zone centrale nécrotique anéchogène

La présentation clinique initiale est souvent une bourse volumineuse, mais non douloureuse, associée à une hydrocèle réactionnelle dans $55 \%$ des cas ${ }^{5}$, d'où la recommandation de rechercher attentivement une lésion tissulaire intra ou paratesticulaire afin d'éviter toute surprise diagnostique à l'intervention. Dans $30 \%$ des cas, la présentation est une masse paratesticulaire.

La plupart des patients ayant eu des mésothéliomes de la tunique vaginale $\mathrm{n}^{\prime}$ ont pas déclaré d'exposition personnelle ni familiale à l'amiante : cette exposition a été signalée dans seulement de $30 \%$ à $40 \%$ des cas. L'atteinte est bilatérale dans $3,8 \%$ des cas ${ }^{1}$.

Le mésothéliome paratesticulaire est souvent diagnostiqué initialement comme tumeur testiculaire ou hydrocèle, mais peut prendre parfois l'aspect d'une épididymite, $d^{\prime}$ 'une hernie inguino-scrotale, $d$ 'une tumeur adénomatoïde ou d'une spermatocèle ${ }^{6}$. Dans le cas de notre patient, les aspects clinique et échographique penchaient en faveur d'une tumeur testiculaire.

Cet aspect échographique atypique a été signalé également par Wolanske ${ }^{7}$, qui a décrit un aspect échographique atypique d'une masse intratesticulaire au niveau du pôle supérieur du testicule droit, sans hydrocèle, et qui s'est révélée par la suite être un mésothéliome malin de la tunique vaginale, comme c'était le cas chez notre patient.

Le diagnostic différentiel doit se faire avec les tumeurs bénignes et les lésions mésothéliales non néoplasiques, comme les tumeurs adénomatoïdes (qui sont de type bénin en présence d'un mésothéliome non papillaire) ou les kystes de la tunique albuginée et du parenchyme testiculaire. L'adénocarcinome du rete testis, le cystadénome papillaire épididymaire et même les métastases testiculaires doivent aussi être considérés comme des diagnostics différentiels ${ }^{8}$.

Les mésothéliomes peuvent être divisés en trois groupes: les mésothéliomes épithéliaux, les mésothéliomes mésenchymateux et les mésothéliomes mixtes. Dans la tunique vaginale, le sous-type prédominant est le mésothéliome épi-

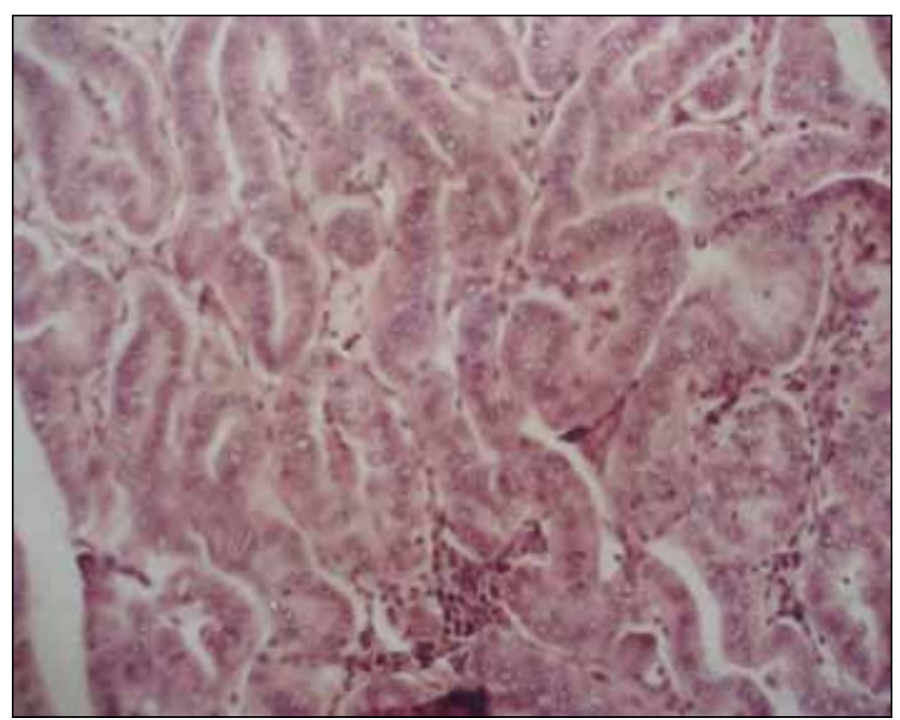

Fig. 2. Aspect histologique d'un mésothéliome de type épithélioïde (grossissement $x 4$ avec coloration à l'hématéine éosine safran)

thélial (50\%-75\%), suivi des sous-types mixte (25\%-35\%) et mésenchymateux (8\%). À l'immunohistochimie, ils sont positifs à la cytokératine, à la kallicréine, à la vimentine et au facteur VIII.

II n'y a pas de coloration avec le CEA, la Leu-M1 ou la cytokératine 20. Le marquage au p53 peut être soit positif soit négatif, sans signification clinique connue ${ }^{9}$.

Ces tumeurs se propagent d'abord aux ganglions lymphatiques, tandis que la diffusion hématogène est moins courante ${ }^{1}$. Les métastases à distance (poumon, foie et plèvre) sont les dernières étapes de la maladie. Il est donc conseillé d'effectuer un bilan d'extension tomodensitométrique afin de détecter d'éventuelles métastases systémiques.

Le traitement curatif est possible dans les premiers stades. Il consiste en une orchidectomie radicale avec excision en bloc de la vaginale. Dans notre cas, et vu le diagnostic initial préopératoire de tumeur testiculaire, l'orchidectomie a été complétée dans un deuxième temps par une hémiscrotectomie afin d'éviter un reliquat tumoral paratesticulaire. II est aussi obligatoire, en cas d'hydrocélectomie et de diagnostic histologique de malignité, de réséquer le tissu cicatriciel scrotal. Une chirurgie conservatrice n'est pas envisageable en raison de l'incidence élevée de récidive. La réponse à la chimiothérapie et à la radiothérapie est très faible.

Le pronostic global est sombre, avec une médiane de survie de 23 mois, mais il est meilleur qu'en cas de mésothéliome pleural ou péritonéal. Après un recul de 2 ans à partir du diagnostic, $60 \%$ des patients présentent des récidives tumorales et $30 \%$ décèdent $^{3}$. Un suivi régulier est donc préconisé afin de déceler les récidives. Le délai de survie diminue à 14 mois en cas de récidive de la tumeur. Il est légèrement plus élevé pour les patients âgés de moins de 60 ans $(p<0,01)$ et au stade localisé du cancer $(p<0,05)^{1,3}$. 


\section{Conclusion}

Le mésothéliome paratesticulaire peut prendre l'aspect d'une masse intratesticulaire. Le traitement consiste à pratiquer une orchidectomie avec résection en bloc de la vaginale. Si celle-ci n'a pas été faite, il est obligatoire de compléter I'intervention par une hémiscrotectomie. Le pronostic est sombre, mais meilleur si le cancer en est au stade localisé, comme ce fut le cas pour notre patient.

Competing interests: The authors all declare no competing financial or personal interests.

This paper has been peer-reviewed.

\section{Références}

1. Plas E, Riedl C, Pfluer H. Malignant mesothelioma of the tunica vaginalis testis. Cancer 1998:83:243746. http://dx.doi.org/10.1002/(SICI) 1097-0142(19981215)83:12<2437::AID-CNCR6>3.0.C0;2-G
2. Barbera V, Rubino M. Papillary mesothelioma of the tunica vaginalis. Cancer 1957;10:183-9. http:// dx.doi.org/10.1002/1097-0142(195701/02) 10:1<183::AID-CNCR2820100127>3.0.C0;2-1

3. Bisceglia M, Dor DB, Carosi I, et al. Paratesticular mesothelioma. Report of a case with comprehensive review of the literature. Adv Anat Pathol 2010;17:53-70.

4. Kossow AS, Mccann LS. Malignant mesothelioma of the testicular tunic. J Urol 1981;26:272-4.

5. Busto Martin L, Portela Pereira P, Sacristan Lista F, et al. Meso-thelioma of the tunica vaginalis. Case report. Arch Esp Urol 2013;66:384-8.

6. Jones MA, Young RH, Scully RE. Malignant mesothelioma of the tunica vaginalis. Clinicopatholigic analysis of 11 cases with review of the literature. Am J Surg Pathol 1995;19:815-25. http://dx.doi. org/10.1097/00000478-199507000-00010

7. Wolanske K, Nino-Murcia M. Malignant mesothelioma of the tu-nica vaginalis testis: Atypical sonographic appearance. J Ultrasound Med 2001;20:69-72.

8. Ulbright TM, Young RH. Metastatic carcinoma of the testis: A clinicopatholic analysis of 26 non incidental cases with emphasis on deceptive features. Am I Surg Pathol 2008;32:1683-93. http://dx.doi. org/10.1097/PAS.0b013e3181788516

9. Churg A. Paratesticular mesothelial proliferations. Sem Diag Path 2003;20:272-8. http://dx.doi. org/10.1053/i.semdp.2003.08.003

Correspondence: Dr. Imad Ziouziou, Service d'urologie B, CHU Ibn Sina, Faculté de médecine et de pharmacie, Université Mohamed V, Rabat, Morocco; imadziouziou@hotmail.com 